\title{
Biochemical, genetic and molecular advances of fragrance characteristics in rice
}

\begin{abstract}
One of the most valuable traits in high-quality rice is aroma or fragrance, which is important for consumer preference and global trade. Aromatic rice is unique and recognized as a badge of honor and an asset in many countries. Among more than 100 volatile components, 2acetyl-1-pyrroline (2AP) is believed to be the main aromatic compound in rice. The principal gene contributing to $2 \mathrm{AP}$ is badh2, which was mapped on chromosome 8 by map-based cloning. A deletion in this gene truncates and makes non-functional the BADH2 protein. Thus, the mutant badh 2 transcript leads to $2 \mathrm{AP}$ accumulation in aromatic rice. The discovery of the gene has led to the clarification of the biochemistry, molecular genetics and evolution of fragrant rice. The breeding of fragrant rice is now faster because of marker assisted selection (MAS), which is based on recognized genes. For a more extensive elucidation of all effective and fundamental factors contributing to rice fragrance, it is essential to further explore target quantitative trait loci (QTLs) and their inheritance and locations.
\end{abstract}

Keyword: Biochemistry pathway; Marker assisted selection; Molecular markers; Quantitative trait loci; Rice aroma 